Review

\title{
Suggested Improvements for Measurement of Equilibrium Solubility-pH of Ionizable Drugs
}

\author{
Alex Avdeef \\ In-ADME Research, 1732 First Avenue \#102, New York, NY 10128 USA \\ Corresponding Author: E-mail: Alex@in-ADME.com; Tel.: +1-646-678-5713 \\ Received: June 14, 2015; Revised: June 22, 2015; Published: July 01, 2015
}

\begin{abstract}
The accurate prediction of solubility of drug-like molecules is difficult, and perhaps a satisfactory general model is not yet available. The most cited challenge to good prediction has been the lack of enough highquality and drug-relevant solubility data that adequately cover the chemical space of drugs. This review addresses data quality in solubility measurement. Specifically, the "gold standard" shake-flask and related methods used to measure equilibrium solubility of ionizable drug-like compounds as a function of $\mathrm{pH}$ were reviewed. Over 800 publications were examined. Many factors affecting the quality of the measurement were recognized, and a number of suggestions are offered to improve the experimental methodology. Some of the suggestions focus on improving methods for future measurements, and some refer to improvements in data mining, i.e., to ways of extracting more reliable information from existing data. By normalizing data for $\mathrm{pH}$ (i.e., deriving intrinsic solubility, $S_{0}$ ) and for temperature (by transforming measurements performed in the range $20-40{ }^{\circ} \mathrm{C}$ to $25{ }^{\circ} \mathrm{C}$ ), it is suggested that the 0.6-0.7 log unit currently expected interlaboratory reproducibility can be reduced to near 0.15 . It is the aim of the review that the improvements in data quality would lead to better predictions of drug solubility using in silico methods.
\end{abstract}

\section{Keywords}

Shake-flask solubility; intrinsic solubility; Henderson-Hasselbalch equation; aggregates; oligomers; micelles.

\section{Introduction}

Since the mid-1990s there has been a heightened effort in drug discovery to predict drug-relevant aqueous solubility, described in at least a hundred publications (e.g., Huuskonen et al. [1,2]; Abraham and Le [3]; Jorgensen and Duffy [4,5]; Bergström et al. [6]; Hou et al. [7]; Delaney [8]; Dearden [9]; Balakin et al. [10]; Taskinen and Norinder [11]; Jain and Yalkowsky [12]; Shayanfar and Jouyban [13]; Wang and Hou [14]; Elder and Holm [15]; McDonagh et al. [16]). The typical errors in drug solubility prediction are $0.7-1.0$ log unit, and for low-soluble compounds, errors are considerably greater than a log unit (Jorgensen and Duffy [5]; Palmer and Mitchell [17]). According to Faller and Ertl [18], whose sentiment may be broadly shared, "no really satisfactory approach to (drug) solubility prediction is available yet," in spite of the large number of prediction studies.

Two large aqueous solubility databases have been the sources for many of the in silico studies. First, the 1608-page Handbook of Aqueous Solubility Data, Second Edition (Yalkowsky et al. [19]) contains over 
18,000 solubility values covering 4661 molecules, collected from many chemical classes, including pharmaceuticals. Second, the PHYSPROP database (Howard and Meylan $[20,21]$ ) from Syracuse Research Corp. (http://www.srcinc.com/) had accumulated over 6000 measured solubility values, with substantial coverage of agrochemicals and potential environmental pollutants. In the two curated compilations, the $\mathrm{pH}$ of saturated solutions had not been reported for most of the ionizable compounds. In addition, smaller databases of crystalline drug-like molecules have been published (Analytical Profiles of Drug Substances [22], McFarland et al. [23]; Rytting et al. [24]; Bergström et al. [25]; Faller and Ertl [18]; Llinàs et al. [26]; Hopfinger et al. [27]). These smaller databases consist largely of intrinsic solubility values, $S_{0}-$ i.e., the solubility of the neutral species.

The most cited challenge to good prediction has been the difficulty to access enough high-quality, drugrelevant, and sufficiently-diverse solubility data that adequately cover the chemical space of drugs and hopefully that of research compounds. In this context, the focus on drug molecules is important, since it is consistently shown that the best prediction models are devised from training sets most similar to the test sets (Walters [28]). However, Palmer and Mitchell [17] posed a contrarian view that the challenge to good prediction might rest in the deficiency of current QSPR methods.

Measuring high quality data is expensive and analytical-resource consuming. Even with great costing, quality is not ensured when results are determined from poorly-designed assays. Selecting training set molecules which are ionizable, but ignoring the effect of $\mathrm{pH}$, can mitigate accurate prediction. Drawing on data from a range of temperatures without adjustments also can be problematic. There are other factors that affect data quality.

To address many of the above concerns, this review draws on our past experiences and also on the examination of over 800 publications to suggest ways (a) to improve the quality of future measurement of equilibrium solubility and (b) to normalize existing data for $\mathrm{pH}$ and temperature effects to extract intrinsic solubility values with improved accuracy. We focus on the shake-flask solubility measurement as a function of $\mathrm{pH}$ which is still the "gold standard" methodology in the minds of most experimentalists. Also, two potentiometric methods are considered. The characterization of solid forms (crystalline, amorphous, nanoparticle, etc.) and their impact on the measured solubility are beyond the scope of this review, although some aspects (i.e., solvate, polymorph, racemate effects) are noted.

In part, this review serves as background preparation for the "panel of experts" solubility session at the 4th World Conference on Physico Chemical Methods in Drug Discovery and Development (PCMDDD-4) in Croatia, 21-24 September 2015 (http://www.iapchem.org/page.php?page id=56).

\section{Methods}

Briefly stated, in the course of gathering published aqueous solubility training data, 803 publications have been examined and a largely fresh intrinsic solubility database containing 4557 entries for 2413 compounds has been assembled. In addition to this, 666 publications reporting $\mathrm{p} K_{\mathrm{a}}$ values have been processed, to add to the in-ADME Research Wiki-p $K_{\mathrm{a}}$ database, now consisting of 2651 qualified entries (www.in-adme.com/wiki pka.php). It has been nearly a full-time project at in-ADME Research since 2011. The collection of training data is nearing a state suitable to support a new solubility prediction effort, which is planned to be the subject of another publication. We are energized to improve the accuracy of the prediction of intrinsic solubility from 2D structure, particularly of sparingly-soluble (or practically-insoluble) ionizable drug-relevant molecules. Preliminary Random Forest regression modeling (Walters [28]) has been tried using the 193 descriptors calculated by the open-source chemoinformatics and machine learning 
RDKit library of programs (Landrum et al. [29]; http://rdkit.readthedocs.org/en/latest/), combined with the Abraham five solvation descriptors (Abraham et al. [30]), and Lang and Bradley [31] predicted melting points in the QsarDB open repository of data and prediction tools (http://qsardb.org/repository/handle/10967/104). The most sensitive molecular descriptors will be further tested in Partial Least Squares models, to better understand the impact of specific descriptors on the predicted solubility.

In the database construction, intrinsic solubility $\left(S_{0}\right)$ values were mostly derived from (a) $S_{w}$ aqueous solubility determined in distilled water, often with $\mathrm{pH}$ not reported, (b) $S_{\mathrm{pH}}$ single-pH buffer values, and (c) multiple-pH buffer $S_{p H}$ values ( $\log S$ vs. $p H$ ). In cases (a) and (b), the Henderson-Hasselbalch equation (discussed below) was assumed to be valid. The pDISOL-X program (in-ADME Research; www.in-adme.com/pdisol $x . h t m l$ ) was used to determine the $S_{0}$ values from the reported $S_{\mathrm{w}}$ and $S_{\mathrm{pH}}$ data, as described in Völgyi et al. [32] and Avdeef [33,34].

Data

The 4557 set of measured solubility values used to deduce the intrinsic solubility $\left(S_{0}\right)$ database were collected from four secondary sources $(57 \%)$ and the rest from primary sources (43\%):

- PHYSPROP database (Sep. 1999 version: 6356 measured water solubility, $S_{w}$ ) [20,21]: 1327 shake-flask values were selected of molecules which were not appreciably ionized at $\mathrm{pH}^{\sim 7}$. Filters used to exclude compounds were: (a) melting point $<40{ }^{\circ} \mathrm{C}$, (b) $\log S_{w}<-8$ or $>0$, (c) surfactants or compounds with long aliphatic chains, (d) multi-ring aromatic hydrocarbons, (e) peroxides, (f) carboxylic acids, (g) salts/complexes with chloride, bromide, iodide, sulfate, phosphate, sodium, potassium, lithium, calcium, magnesium, zinc, copper, arsenic, mercury, lead, antimony, silver, silicon, tin, etc., (h) dyes or names containing color, and (i) herbicides, pesticides, insecticides, rodenticides, and acaricides (as indicated by "tags" at the Royal Society of Chemistry ChemSpider website: http://www.chemspider.com/). Of the selected compounds, the $S_{w}$ values of 1210 nonionizable/nonionized molecules were taken to be $S_{0}$. The remaining 117 compounds were processed by $\mathrm{pDISOL-X}$ to calculate $S_{0}$ and $\mathrm{pH}_{\text {sat }}(\mathrm{pH}$ of saturated aqueous solution) from the given $S_{\mathrm{w}}$ and $\mathrm{p} K_{\mathrm{a}}$.

- Handbook of Aqueous Solubility Data [19]: $1130 S_{w}$ data were selected, with 776 values subjected to $p$ DISOL-X analysis to determine $S_{0}$ values. A few of the same preceding filters were used in the selections.

- Analytical Profiles of Drug Substances [22]: All 39 volumes of the series of monographs were searched for quantitative solubility data. Monographs on 155 molecules were found to be useful. Most of the reported solubility values of ionizable molecules were measured in pure water with unspecified saturation $\mathrm{pH}$. For those ionizable molecules which were not salts, the intrinsic values were calculated by $p$ DISOL-X. Unfortunately, the solubility accompanied scant experimental detail (e.g., temperature not always reported), and many entries were referenced as 'personal communication.' But there are several high-quality $\log \mathrm{S}-\mathrm{pH}$ data sets in the monographs.

- Miscellaneous publications (shake-flask data, some from secondary sources): $852 S_{0}$ values (taken as reported).

- CheqSol $S_{0}$ Data (potentiometric): 201 values for 151 molecules collected from several publications (Stuart and Box [35]; Sköld et al. [36]; Llinàs et al. [26,37]; Box et al. [38]; Hopfinger et al. [27]; Narasimham et al. [39]; Hsieh et al. [40]; Comer et al. [41]; Palmer and Mitchell [17]; Schönherr et al. [42]). 
- Dissolution Template Titration (DTT) $S_{0}$ Data (potentiometric): 75 published values were collected (Avdeef [43,46]; Avdeef et al. [44]; Avdeef and Berger [45]; Faller and Wohnsland [47]; Bergström et al. [48]; Fioritto et al. [49]; Ottaviani et al. [50]).

- Rytting et al. [24] free-base and -acid (no salts used) shake-flask $S_{w}$ : solubility of 122 molecules were gathered, all measured in one laboratory, with $S_{0}$ calculated by $p D I S O L-X$.

- Shake-flask measurements in two or more pH buffers (primary sources): 697 molecules with S-pH data were analyzed by $p$ DISOL-X to determine $S_{0}$. Figure 1a shows the frequency distribution of the multi-pH data. There were 101 studies (14\%) with 2 pH/assay and 164 studies (24\%) with 3-5 pH/assay. Eight studies were reported, each with more than $40 \mathrm{pH}$ points defining the log $\mathrm{S}$-pH profile. The median in the set of 697 compounds was seven S-pH points per molecule. Most of the published data were presented graphically as $\log S$ vs. $\mathrm{pH}$ plots. The process of digitizing data from plots introduced some small error. It was not possible to digitize the graphs entirely if the molecules were low soluble and the plots were in non-logarithmic $S$ units vs. $\mathrm{pH}$. A large fraction of the primary source data originated from five journals (in rank order): Int. J. Pharm., J. Pharm. Sci., Pharm. Res., J. Chem. Eng. Data, and Eur. J. Pharm. Sci. It is primarily from this group of 697 "gold standard" shake-flask multi-pH measurements that many of the suggestions below were formulated.

The assembled 4557 intrinsic solubility set ranges in $\log S_{0}$ from -11.6 to +1.8 (log molarity). Figure $1 \mathrm{~b}$ shows a frequency distribution for the set. About $40 \%$ of the compounds have log $S_{0}$ between -6 and -3 , the typical range of values for research compounds (Faller and Ertl [18]; Walters [28]). The median $\log S_{0}$ of the distribution is -2.8 . About $5 \%$ of the molecules have $\log S_{0}<-6$. The least-soluble molecules $\left(\log S_{0}<-8\right)$ in the set are amiodarone, cosalane, halofantrine, clofazimine, itraconazole, quinclorac, probucol, brodifacoum, epristeride, silafluofen, carbenoxolone, tamoxifen, fluotrimazole, moteretinide, esfenvalerate etofenprox, etretinate, and NPC-1161C (which includes four agro-chemicals). The most soluble ( $\left.\log S_{0}>0\right)$ substances are amino acids, simple carboxylic acids, and carbohydrates.

The compounds in the 4557-set are solids at room temperature, with propofol and nitroglycerin at the border line with the melting points 19 and $14{ }^{\circ} \mathrm{C}$, respectively. The shake-flask values were mainly clustered around room and physiological temperatures: $23 \pm 3^{\circ} \mathrm{C}(78 \%)$ and $37 \pm 5^{\circ} \mathrm{C}(22 \%)$. Figure $1 \mathrm{C}$ shows the temperature frequency distribution. Surprisingly, a large number of sources don't state the temperature used in the assay. Contextually, room temperature is a reasonable guess in most instances. Other publications cite "room temperature." Where the precise temperature was not reported, it was taken to be $23^{\circ} \mathrm{C}$ in the assembled database.

Figure 2 shows plots of six high-quality examples of multi-pH solubility data analyzed by $p$ DISOL-X. Most of the compounds can be represented by simple Henderson-Hasselbalch equations (cf., below). The data from verapamil was best fit with a tricationic aggregate below $\mathrm{pH}$ 7. Above $\mathrm{pH} 9$ the free-base form of verapamil may participate in the formation of uncharged water-soluble aggregates or micelle-like structures, consistent with the discussion by Surakitbanharn et al. [51]. 
(a) MULTI-pH SHAKE-FLASK MEASUREMENT

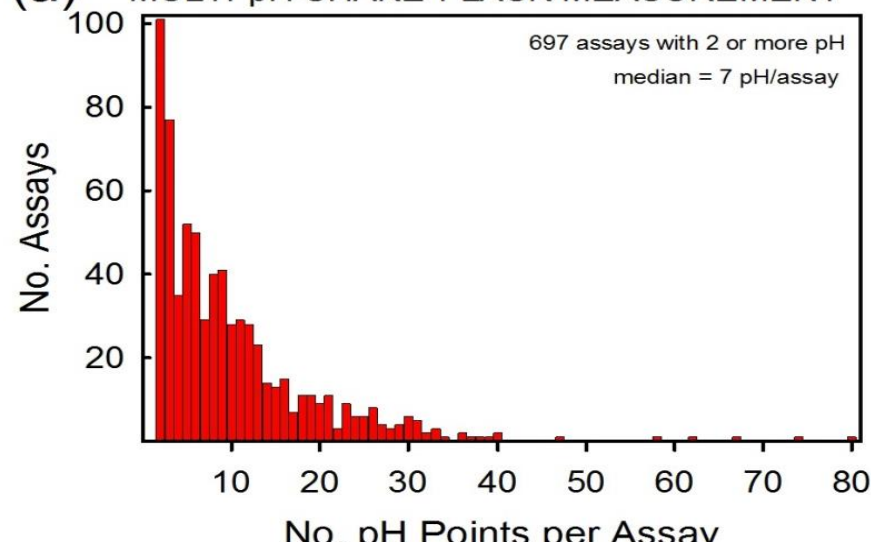

(b) INTRINSIC SOLUBILITY DATABASE

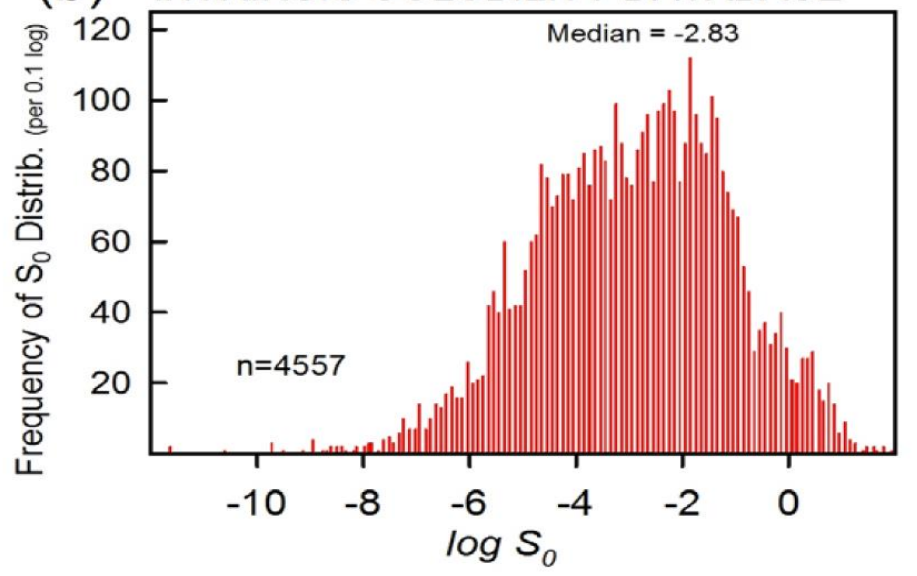

(c) TEMPERATURE DISTRIBUTION

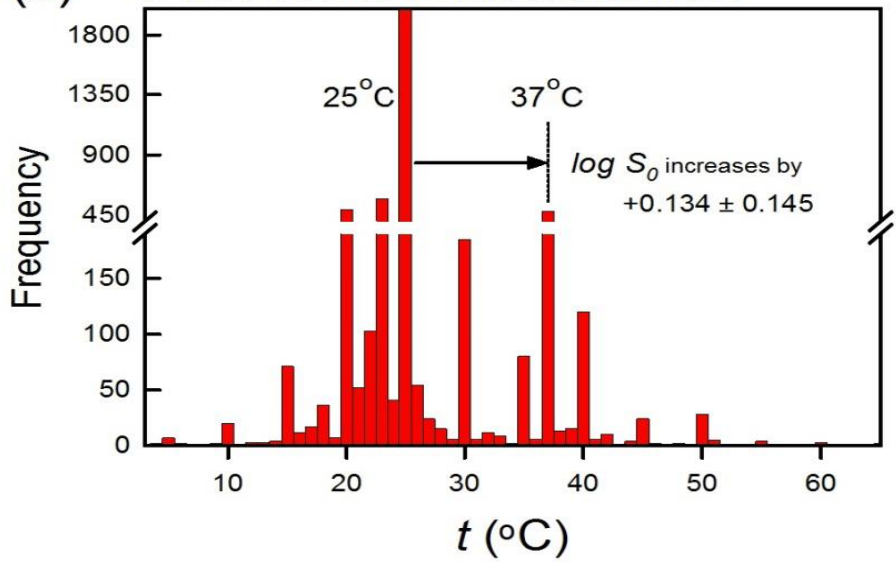

Figure 1. (a) Distribution of the number of multiple-pH shake-flask measurements per assay. (b) Intrinsic solubility, $\log S_{0}$, distribution, as counts in $0.1 \log$ unit intervals. (c) Distribution of assay temperature in the 4557 -set. On the average, $\log S_{0}$ increases by $0.134 \log$ unit as temperature goes from 25 to $37^{\circ} \mathrm{C}$. 

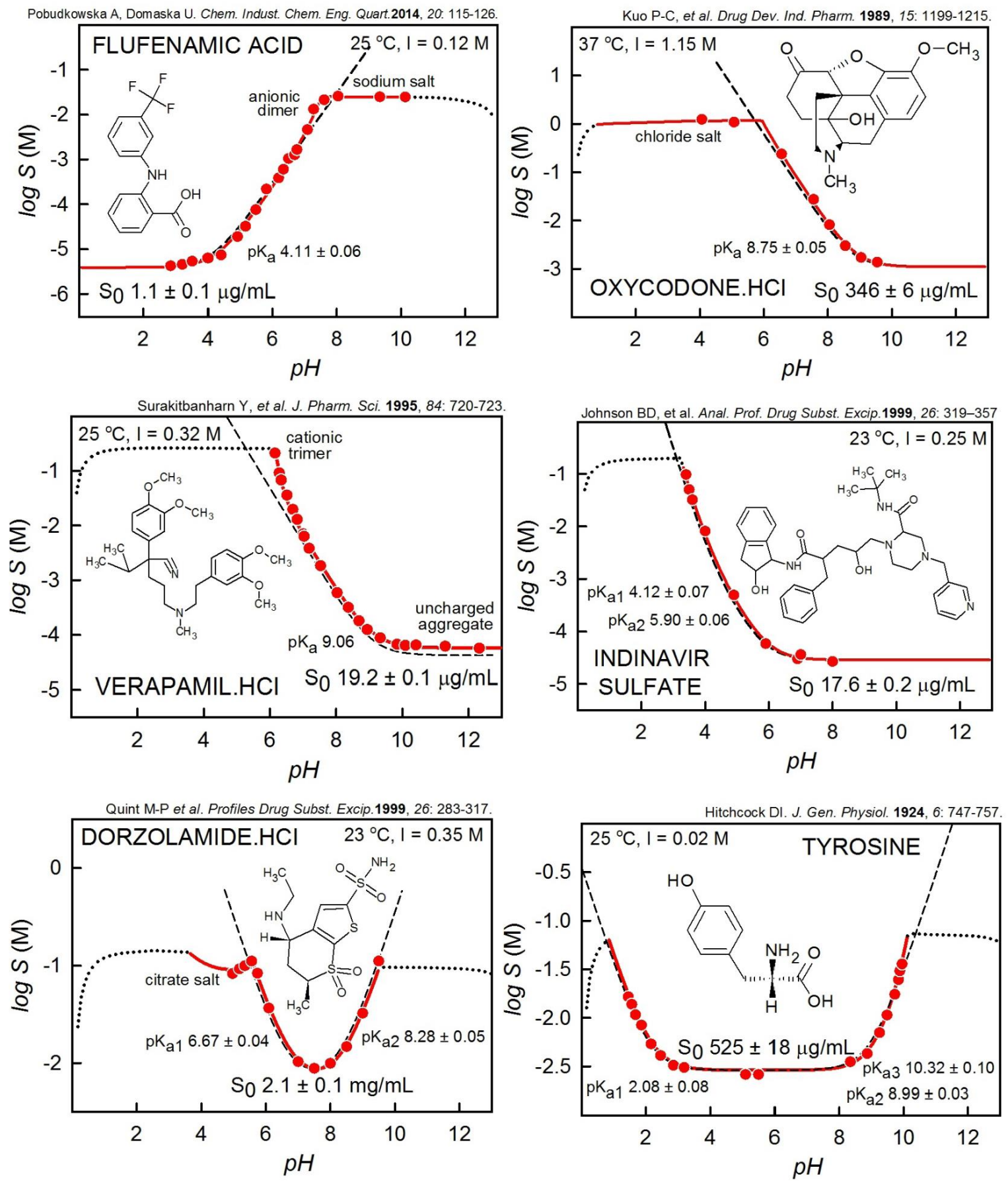

Figure 2. Examples of log $S-\mathrm{pH}$ profiles from well-designed assays for an acid (flufenamic acid), three bases (oxycodone, verapamil, and indinavir), and two ampholytes (dorzolamide and tyrosine). Note that the tyrosine is based on measurements done in 1924. The solid (red) curves represent the best-fit $p$ DISOL-X model which rationalizes the measured solubility values (filled circle symbols). The dotted curves depict the $\mathrm{pH}$ interval where the compounds were fully-dissolved. The dashed curves were calculated from the Henderson-

Hasselbalch equations. Flufenamic acid, oxycodone, and dorzolamide were studied at concentrations high enough to effect salt precipitation. In all cases but verapamil, it was possible to refine the $\mathrm{p} K_{\mathrm{a}}$ values. Verapamil and flufenamic acid show some evidence of formation of aggregates. 


\section{Results and Discussion}

\section{Solubility Units and Conversion Issues}

Solubility measurements have been reported in a multiplicity of concentration units: $\mathrm{mol} / \mathrm{L}$ (molarity, $\mathrm{M}$ ), $\mathrm{mM}, \mu \mathrm{M}, \mathrm{mol} / \mathrm{kg}$ (molality, $\mathrm{m}$ ), mole fraction, mass fraction, weight $/ \mathrm{mL}$ (e.g., $\mathrm{mg} / \mathrm{mL}, \mu \mathrm{g} / \mathrm{mL}, \mathrm{ng} / \mathrm{mL}$, $\mathrm{pg} / \mathrm{mL} \ldots$..), $\mathrm{mg} / 100 \mathrm{~mL}, \mathrm{mg} / \mathrm{dL}, \% \mathrm{w} / \mathrm{v}, \mathrm{g} \% \mathrm{~mL}, \mathrm{mg} / \mathrm{mL} \%, \mathrm{mg} \%$, "1 in 40 of water," "soluble in 2 parts of water," "3\% soluble in water," units of $\mathrm{IU} / \mathrm{mL}$, etc. Mole fraction, mass fraction, and molality units are almost always used when solubility is determined over a wide range of temperatures, since the units do not depend on the density of the solutions. It is not clear why units such as $\mathrm{mg} \%$ would be preferred. In certain publications such units are not explicitly defined. Usually (but not always), mol fractions are precisely defined in the publication, so that conversion to molarity is straight forward if the solution density is known (which is slightly higher than that of pure water). The same cannot be said of the very frequently used weight/mL "practical" units. In the clearly presented papers, the equivalent molecular weight to use to convert the practical values to molarity is stated (e.g., "concentration is expressed as free base equivalent") or is evident, but too often, the reader has to guess what the authors intended. For a given molecule, often the uncertain units became evident when compared to results from different publications.

As different units are in common usage, consequently a useful sense of comparisons between different studies is a challenge. On a practical note, it is all too easy to make a mistake in converting the units to the preferred molarity scale of the database. It could be argued that solubility should be presented in log units (preferably based on molarity), since (a) direct values span over 12 orders of magnitude and cannot be accurately depicted in S-pH plots at the low end of the scale, and (b) since errors in log values do not depend on the magnitude of the log solubility (whereas they do when direct units are considered). For example, it should be possible to claim that shake-flask log $S$ values have an average reproducibility of 0.1 log unit for a given molecule between values reported in different publications; it makes no obvious sense to present an average reproducibility value of $S$ measurements that can span so many orders of magnitude. In the 4557-set database, values reported in molality units were noted, but not converted to those in molarity (by correcting for density), since the differences are small near the temperature range of interest $\left(20-40^{\circ} \mathrm{C}\right)$, and the since the actual solution density is seldom reported.

\section{Impact of Accuracy in the Type of Data used for Prediction Training Sets}

Most studies aimed to predict $S_{0}$ values, whether explicitly stated or not. However, most studies used $S_{w}$ values in the training sets, which resulted in increased errors for low soluble ionizable molecules. The exception is with nonionizable molecules, where water and intrinsic solubility are the same: $S_{w}=S_{0}$.

The majority of the aqueous solubility values, $S_{w}$, were measured by adding excess solid (ideally, the free-acid or free-base, not the salt) to distilled water. Frequently, $\mathrm{pH}$ of the saturated solution, $\mathrm{pH}_{\text {sat, }}$ was not reported (and probably not measured).

When a moderately soluble weak acid or base is added to distilled water, the aqueous $\mathrm{pH}$ is altered by the ionizing molecule, in the direction where the molecule remains largely in the uncharged form: $S_{w} \approx S_{0}$, provided the compound is added as a pure free acid or free base. For compounds added as salts, it is frequently not possible to deduce $S_{0}$ and $\mathrm{pH}_{\text {sat }}$ from just $S_{\mathrm{w}}$ and $\mathrm{p} K_{\mathrm{a}}$, since the total added amount of compound can affect the disposition of the saturated solution. For example, if not enough salt form of the 
drug is added, the solid disproportionates to the free acid/base in the saturated solution, with $\mathrm{pH}_{\text {sat }}$ depending on the weight of salt added.

If the ionizable compound is practically insoluble, then the value of $S_{w}$ can be quite different from $S_{0}$, since not enough of the compound dissolves to alter the $\mathrm{pH}$ in the direction of maintaining a nearly uncharged molecule. In such cases, it is possible to calculate the $\mathrm{pH}_{\text {sat }}$, as well as $S_{0}$, provided the $\mathrm{p} K_{\mathrm{a}}$ is known and that the Henderson-Hasselbalch equation accurately describes the solubility-pH relationship for a one-p $K_{\mathrm{a}}$ molecule (' \pm ' in Eq. 1 : acid when ' + ' and base when ' - '):

$$
\log S=\log S_{0}+\log \left\{10^{ \pm(\mathrm{pH}-\mathrm{pKa})}+1\right\}
$$

More complex Henderson-Hasselbalch equations for ampholytes and multiprotic acids and bases have been tabulated elsewhere [52,57].

A feature of $p$ DISOL-X allows the calculation of $\mathrm{pH}_{\text {sat }}$ : when the $\mathrm{pH}$ is not reported with the $\mathrm{S}_{\mathrm{w}}$ value, a $\mathrm{pH}_{\text {sat }}$ of 7.0 is initially assumed, and the regular mass balance regression analysis is performed [32]. (Fixed values of carbon dioxide may be included.) The $\mathrm{pH}$ titrant volume is calculated. If the volume is non-zero, the value of $\mathrm{pH}_{\text {sat }}$ is adjusted iteratively by the regression procedure until the calculated volume is driven to zero. At the same time, $\mathrm{S}_{0}$ is refined.

\section{Variance Increase due to Pooling of Training Set Solubility Data Determined at Different Temperatures}

It appears that many of the published prediction studies have drawn data from two large secondary sources, as noted above. These two compilations have data collected mostly in the range $20-40{ }^{\circ} \mathrm{C}$, roughly in a bimodal distribution (cf., Fig. 1c). Some prediction papers state that only $25^{\circ} \mathrm{C}$ data were used. Many prediction papers are less clear, and it might be that some pooling from different temperatures takes place, which would contribute to increased variance in the experimental training set data. If the temperature dependence of solubility could be predicted, then the number of available training set values in the former case would increase, and in the latter case the variance in the measured data would decrease. That is, solubility values could be normalized to a single reference temperature, e.g., $25^{\circ} \mathrm{C}$. As far as we are aware, there are no publications where temperature dependence of solubility is predicted solely from 2D structures. We have collected a database of 626 values of enthalpies of solution, with 77 determined by calorimetric methods, and the rest by solubility methods (van't Hoff slopes from log $S$ vs. $1 / T$ plots). The calorimetric data are producing the most promising results, with $r^{2}>0.5$ using just the Abraham solvation descriptors. This procedure will be described in a separate publication.

As a preliminary observation, on the average, measured log $S_{0}$ values increase by $0.13 \log$ unit (cf., Fig. 1c), as the temperature is raised from 25 to $37^{\circ} \mathrm{C}$. In cases where the training set data are pooled from multiple temperatures, the variance can be expected to increase by about 0.13 log unit. This can be avoided if the data mining process were to convert measurements to a single reference temperature.

\section{Limitations of Intrinsic Solubility, $S_{0}$, Determined from a Single Measurement of $S_{w}$}

When measured compounds contain protogenic impurities, the $\mathrm{pH}_{\text {sat }}$ may be affected, which could lead to a change in the measured $S_{\mathrm{w}}$. For this reason, it is highly recommended that $\mathrm{pH}_{\text {sat }}$ be measured and not just calculated. Otherwise, the conversion of $S_{w}$ to $S_{0}$ may be inaccurate. 
Abraham and Le [3] discussed the relationship between the measured $S_{w}$ and the underlying $S_{0}$ for ionizable molecules, and identified under which circumstances large errors could result for $S_{\mathrm{w}}$ used (in place of the calculated $S_{0}$ ) as the training set values. The authors derived useful plots of $S_{0} / S_{w} v s . \log S_{w}$ for acids and bases over a range of $\mathrm{p} K_{\mathrm{a}}$ values. For example, $S_{\mathrm{w}} \approx S_{0}$ for acids with $\mathrm{p} K_{\mathrm{a}} 5$ and $S_{\mathrm{w}}>0.001 \mathrm{M}$, or with $\mathrm{p} K_{\mathrm{a}} 3$ and $S_{\mathrm{w}}>0.1 \mathrm{M}$. With bases, $S_{\mathrm{w}} \approx S_{0}$ for $\mathrm{p} K_{\mathrm{a}} 10$ with $S_{\mathrm{w}}>0.01 \mathrm{M}$, or $\mathrm{p} K_{\mathrm{a}} 8$ with $S_{\mathrm{w}}>0.0001 \mathrm{M}$.

There are further considerations. When the aqueous solubility of practically insoluble free bases ( $\mathrm{p} K_{\mathrm{a}}>$ 9) are measured in distilled water, the $\mathrm{pH}$ is only slightly affected by the extent to which the base dissolves. Most notably, the observed $\mathrm{pH}$ often is regulated by a much stronger buffer present in water: ambient dissolved carbon dioxide. This is often overlooked. The $\mathrm{pH}$ of the drug-saturated solution can vary from 610, depending on how much $\mathrm{CO}_{2}$ is dissolved in water and how insoluble the basic compound is. For example, Rytting et al. [24] reported $\log S_{\mathrm{w}}=-4.87$ (molarity) for terfenadine dissolved in water. If $\mathrm{CO}_{2}$ content in solution were ignored, the calculated $\log S_{0}=-5.7$ and $\mathrm{pH}_{\text {sat }}=9.2$. However, if $\left[\mathrm{CO}_{2}\right]=20 \mu \mathrm{M}$ (a common ambient level), the calculated $\log S_{0}=-8.3$ and $\mathrm{pH}_{\text {sat }}=6.6$. The error in determining $S_{0}$ of molecules like terfenadine in distilled water is expected to be enormous (as much as 3 log units), since it is very difficult to eliminate $\mathrm{CO}_{2}$ entirely simply by bubbling an inert gas through solution. In fact, any protogenic drug impurity in solution under the circumstances would lead to large uncertainties in the intrinsic solubility of the drug substance of interest. The simple remedy might be to measure the $\mathrm{pH}$ of the saturated solution. However, measuring the $\mathrm{pH}$ accurately when the solution is essentially unbuffered is problematic, due to the effects of uncontrolled electrode junction potentials and other factors [52].

\section{Added Buffer Improves Accuracy when Measuring Aqueous Solubility $\left(S_{p H}\right)$ at a Single $p H$}

The above terfenadine water solubility example illustrates that high errors can result when the $\mathrm{pH}$ of the saturated solution is not known or whose measurement is problematic. The remedy is to buffer the solution (but not excessively) and actually measure the $\mathrm{pH}$ when the saturated solution reaches equilibrium. However, when only a single aqueous solubility at a known $\mathrm{pH}, S_{\mathrm{pH}}$, is measured, it is still necessary to assume that the Henderson-Hasselbalch equation accurately describes the log $S$ - $\mathrm{pH}$ profile, in order to calculate the $S_{0}$, given an accurate independently-determined $\mathrm{p} K_{\mathrm{a}}$.

\section{Measurement of Aqueous Solubility at Several Buffered $p H$, below and above the $p K_{a}$, for Best Accuracy}

By measuring solubility at several different values of $\mathrm{pH}$ (cf., Fig. 2) below and above the $\mathrm{p} K_{\mathrm{a}}$ of an ionizable molecule, it's possible to overcome several of the above sources of error. The $\log S$ data as a function of $\mathrm{pH}$ can be analyzed, to determine the value of $S_{0}$. The validity of the Henderson-Hasselbalch equation can be easily tested in such analysis, using mass-balance based solubility simulation software (e.g., $p \mathrm{DISOL}-\mathrm{X})$.

A universal buffer mixture (e.g., whose $\mathrm{pH}$ is nearly linearly controlled by added aliquots of standardized $\mathrm{NaOH}$ solution) or several independent buffers may be used, but the $\mathrm{pH}$ still needs to be measured when the saturated solution reaches equilibrium. It can be misleading to assume that the buffer $\mathrm{pH}$ will remain unchanged in the course of the drug dissolution. Nor is it a good idea to use excessively high concentrations of buffers, since drug-buffer complexes and precipitates may form, affecting the measured solubility (Shoghi et al. [53]).

In simply-behaving systems, even the $\mathrm{p} K_{\mathrm{a}}$ can be determined from the log $S-\mathrm{pH}$ data (Zimmermann [54]; cf., Fig. 2), but this is not generally recommended [32-34]. It is far more reliable to use purposedesigned $\mathrm{p} K_{\mathrm{a}}$ measurement techniques (e.g., potentiometric, spectrophotometric, or capillary 
electrophoretic), under conditions where ionic strength is well controlled and complications due to sample complexation, self-aggregation, or precipitation are avoided.

\section{Calibration of $\mathrm{pH}$ Electrode}

Glass $\mathrm{pH}$ electrodes are not all equal. There is no single standard method for calibrating electrodes. In solubility publications, the electrode calibration is virtually never described. The make of the electrode is hardly ever stated. In contrast, researchers who determine $\mathrm{p} K_{\mathrm{a}}$ values using commercial instruments are well acquainted with the routinely-used four-parameter procedure (Avdeef and Bucher [55]). Measurements of $\mathrm{pH}$ are sensitive to ionic strength effects, especially in poorly buffered solutions. In measurements of salt solubility, it is not uncommon to have the ionic strength reach 1-3 M. Methods for electrode calibration to address these challenging conditions have been discussed in the literature (Völgyi et al. [32]; Wang et al. [56]). Errors in the $\mathrm{pH}$ scale can easily exceed 0.1-0.2 units in the mid-scale region. For measurements at $\mathrm{pH}<1$ or $\mathrm{pH}>12$, the $\mathrm{pH}$ scale can be in error by as much as 0.5 log unit. The $\mathrm{pH}$ electrode practices routinely used in modern $\mathrm{p} K_{\mathrm{a}}$ methodology would benefit solubility measurement and lead to improved quality of results.

\section{Separating Solid from Saturated Solution}

An advantage of the potentiometric methods is that they do not require phase separation. Also, certain fiber optic probe methods can determine concentrations in turbid solutions (cf., Appendix). However, most traditional methods require some sort of phase separation.

Of the 4557 entries in database, there were only 583 indications of the type of phase separation used. It's surprising that not all primary sources identified the means of separation, although one might surmise that most of these employed filtration. None of the secondary sources lists such detail. Of the indicated values, $44 \%$ used some means of filtration, $14 \%$ used centrifugation, and $9 \%$ used sedimentation solely. The majority of those using filtration seemed to be aware of the problem of filter adsorption and discarded the initial filtrate solution or performed "double filtration" [52,57]. A few of those using filtration did not take heed of the Chen et al. [58] recommendations against using nylon filters.

A solid drug substance in equilibrium with its saturated solution is dynamically dissolving and precipitating at equal rates. Since that equilibrium is maintained by the presence of the solid, the act of separation by filtration disrupts the process, to the extent that the solution concentrations may be altered by reactions of the substance with the container and filter surfaces. Thus, avoiding filtration, if possible, can be recommended. However, it is such a popular procedure, that most non-potentiometric protocols use it.

Especially noteworthy, $13 \%$ of separations were done by centrifugation, followed by filtration. This can be problematic with low soluble compounds, for the above reasons. If a saturated solution is devoid of excess solid (which centrifugation removes), then passing the weakly-poised (i.e., nearly subsaturated) solution through a filter can produce a significantly subsaturated solution. Vessel surface adsorption may contribute to a further lowering of the amount of dissolved sample. In such a combined procedure, solubility may be significantly underestimated. The above practice is best avoided, especially with low soluble compounds. 
Centrifugation is needed in cases of samples that form (a) opalescent saturated solutions (e.g., colloid systems), or (b) stable suspensions that do not sediment easily, or (c) small agglomerate particulates that can pass through commonly-used filtration membranes. It was shown by McGovern et al. [59] that certain types of low soluble research compounds, dubbed "promiscuous inhibitors," form agglomerates of the order of $0.1 \mu \mathrm{m}$ in size. These can pass through most filters used for phase separation, leading to overestimates of the solubility. If such compounds are suspected, it may be a useful first to filter the suspension and then to ultracentrifuge it (in that order).

Equilibration Time and Identifying the Final Form of the Solid in the Saturated Solution

Most of the equilibrium protocols reviewed here strive to determine the solubility of the most stable form of the solid, most likely crystalline (and sometimes hydrated). Assay times are selected to be long enough to ensure that the measured solubility is no longer changing and that it has reached its lowest value. It is a common practice in pharmaceutical research to adopt $24 \mathrm{~h}$ for the equilibration time ("one-shoe-size-fits-all" approach). In most cases, $24 \mathrm{~h}$ is enough, but certain practically-insoluble compounds, which consequently have very slow dissolution rates may require much longer times [34]. Loftsson et al. [60] allowed 3-7 days for equilibration to be reached for a variety of low soluble compounds. Other researchers used 2-10 days equilibration times for practically-insoluble anticancer drugs (Venkatesh et al. [61]; He et al. [62]). Fini et al. [63] allowed diclofenac suspensions to incubate for 30 days. Undeniably, the stability of the compound needs to be verified when such long times are used.

All too often, at the end of equilibration, the actual form of the solid is not characterized in reported studies. Often the mono- or dihydrate is more stable (i.e., less soluble) than the anhydrous form of the solid. Sometimes, multiple-hydrate forms of the solid precipitate in the crystalline form. But this is not always characterized in published solubility-pH studies. (a) DTT - Shake-Flask COMPARISON

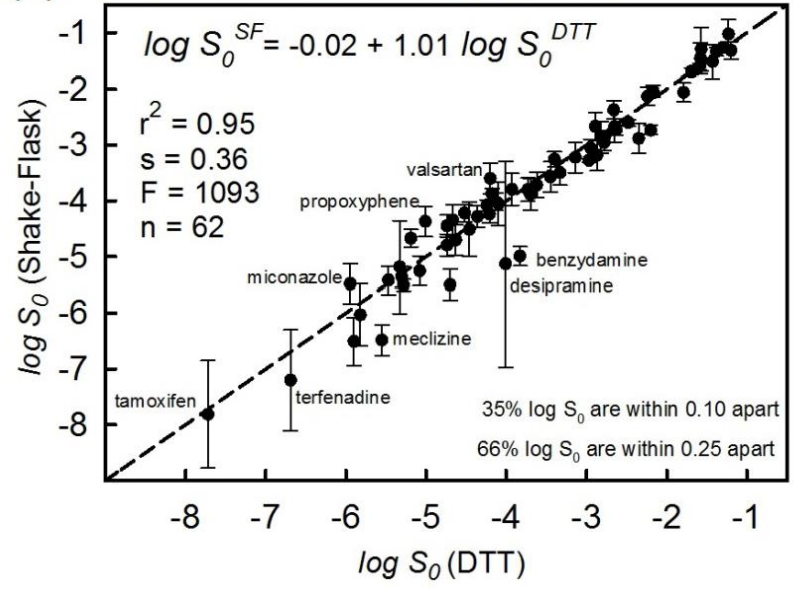

(b) CheqSol - Shake-Flask COMPARISON

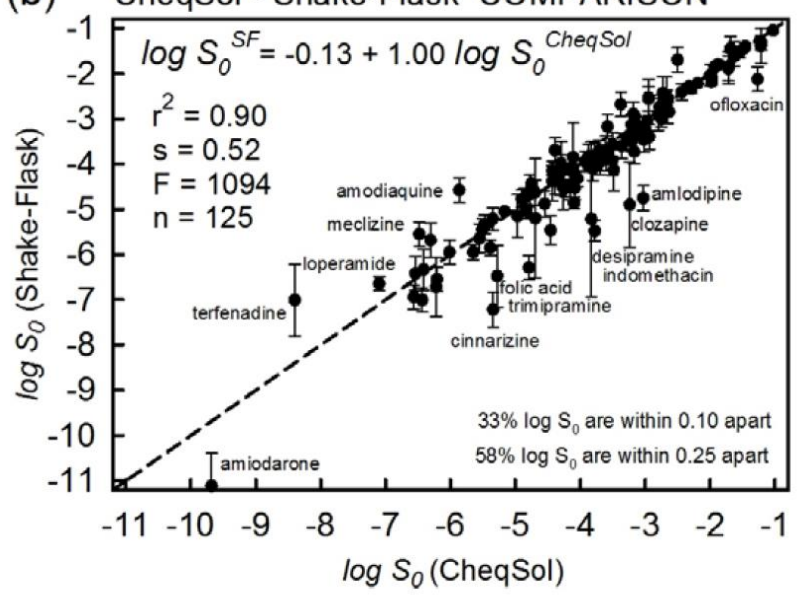

(c) $\mathrm{pH}$-Corr 1-pt $\mathrm{S}_{\mathrm{w}}$ - Shake-Flask COMPARISON

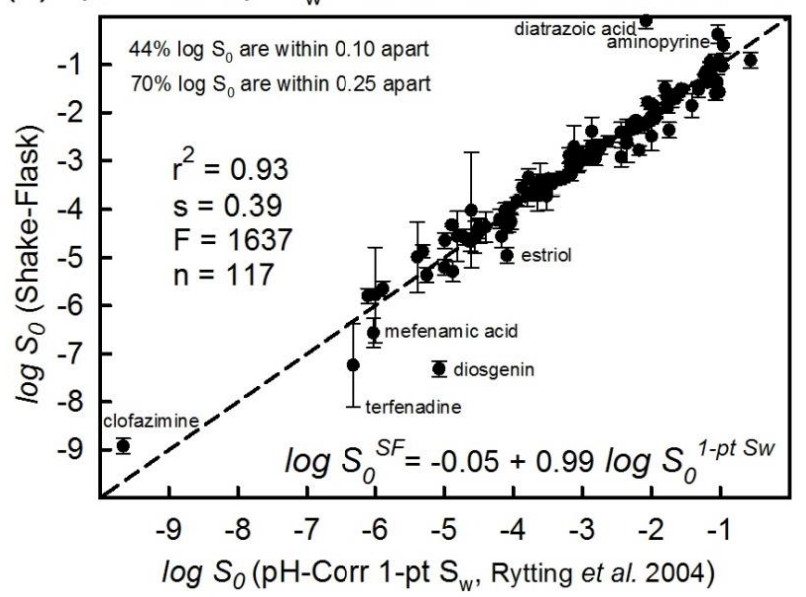

Figure 3. Comparison of traditional shake-flask measurements to (a) the Dissolution Template Titration (DTT) potentiometric method, (b) the CheqSol potentiometric method, and (c) the shake-flask method done in one laboratory using the same protocol. 
Solvates aside, different polymorphs of the same stoichiometry may form, depending on the specific assay protocol. Pudipeddi and Serajuddin [64] examined the differences between the solubility values of polymorphs of various substances and found that $0.3 \mathrm{log}$ unit was a typical spread. A few substances showed differences as high as a log unit.

DL-racemates of optically active molecules can have solubilities lower than those of pure enantiomers. For example, at $25^{\circ} \mathrm{C}$, DL-tartaric acid has $\log S_{0}=+0.18$, but the L-form has +0.58 , an increase of $0.40 \log$ unit. Fourteen amino acids were found with solubility values reported for both the $\mathrm{DL}$ - and L- forms. On the average the $\log S_{0}$ of the DL- enantiomer is 0.15 lower (more insoluble) than that of the L- enantiomer. The least-soluble amino acids (tryptophan, tyrosine, and cystine) show the largest differences in the solubility between the DL- and the L-forms (i.e., DL-forms less soluble). Very few chiral drugs have both DL- and L-/Dform solubilities reported. As an example, DL-atropine has $\log S_{0}=-2.20 \pm 0.03(n=3)$; L-atropine has -1.84 $\pm 0.10(n=2)$, an increase of $0.36 \log$ unit.

When solubility of chiral molecules is reported without mention of the enantiomeric state, racemic mixture is probably implied.

As mentioned above, in many solubility studies, the solid added to solution is in the salt form (chloride, maleate, tartrate, sodium, etc.). The solid that is isolated at the end of equilibration may not be that of the original salt form, but rather of the free-acid/-base. This can lead to ambiguity in ascribing the reported solubility (Anderson and Flora [65]).

Consequently, crystal-form uncertainty translates to higher variances in prediction studies, where many literature values are pooled into the same training set. Molecular descriptors based on 2D considerations may be difficult to assign to test compounds which may exhibit significant polymorphism/solvatomorphism.

\section{Reproducibility of Experimental Solubility Data}

Interlaboratory reproducibility can be assessed for a few model compounds which are repeatedly used in solubility studies. For example, reported solubility of anthracene from 17 different laboratories indicate standard deviation, SD = $0.17 \mathrm{log}$ unit (Kishi and Hashimoto [66]). Additional examples for phenytoin, $\mathrm{SD}=$ $0.15(n=16)$, flurbiprofen, $S D=0.19(n=16)$, and diclofenac, $S D=0.20(n=20)$ show similar reproducibility (this work). Perhaps, these can be the expected interlaboratory reproducibility values for such relatively simple molecules. However, ionizable molecules with very low solubility might be expected to have poorer reproducibility. Particularly poignant examples are indicated (this work) by indomethacin (which can be unstable at elevated $\mathrm{pH}), \mathrm{SD}=0.79(\mathrm{n}=12)$, and terfenadine (whose unbuffered $S_{\mathrm{w}}$ is strongly affected by ambient $\left.\mathrm{CO}_{2}\right), \mathrm{SD}=1.48(\mathrm{n}=12)$. The Handbook of Aqueous Solubility Data [19] is a massive source of values to estimate interlaboratory reproducibility, although a systematic analysis of the compiled data has not been published, as far as we are aware. For 411 compounds with reported replicate measurements, Katritzky et al. [67] found average SD $=0.58$. Comparable values have been reported for research compounds. According to Taskinen and Norinder [11], an AstraZeneca in-house database of solubility measurements of different batches of the same compound typically showed reproducibility of $0.49 \mathrm{log}$ units. Higher uncertainties had been suggested (Jorgensen and Duffy [5]; Palmer and Mitchell [17]).

However, there are many compounds determined in different laboratories that indicate reproducibility much less than the above SD values. For example, barbital, hydrochlorothiazide, and lidocaine show SD = $0.08 \log$ unit for $n=10-14$ each; for hydrocortisone $S D=0.07(n=11)$; for testosterone $S D=0.06(n=10)$, and for acetanilide, aminopyrine, threonine, alanine, 5-fluorouracil, budesonide, minoxidil, fluconazole, 
corticosterone, phenobarbital, lidocaine, hydroflumethiazide, acetaminophen, serine, glycine, atenolol, SD $=0.01-0.05(n=4-11)$.

In this review of shake-flask solubility measurement, it was decided to include data from two potentiometric methods (DTT and CheqSol - c.f., Appendix). It was thus of interest to estimate how concordant the DTT and CheqSol data are with shake-flask (SF) results for molecules evaluated by the different approaches from different laboratories.

Figure 3a shows a correlation plot of $\log S_{0}$ (SF) vs. $\log S_{0}$ (DTT). Although some replicate SF measurements possess large variances, the two sets of $\log S_{0}$ values correlated well: $\log S_{0}^{\mathrm{SF}}=-0.02+1.01$ $\log S_{0}{ }^{\mathrm{DTT}}, \mathrm{r}^{2}=0.95, \mathrm{~s}=0.36, \mathrm{~F}=1093, \mathrm{n}=62$. Based on $\mathrm{SF}$ replicates, the average $\mathrm{SD}=0.27$, which is not very different for the correlation standard deviation, $s=0.36$. The differences between the two log $S_{0}$ sets were $\leq 0.25$ for $66 \%$ of the molecules. A weighted linear regression, using the individual inverse variances of the SF data as weights did not appreciable change the statistics of the SF - DTT comparison.

Figure $3 \mathrm{~b}$ shows a plot comparing SF to CheqSol measurements, indicating that the two sets appear comparable: $\log S_{0}{ }^{\mathrm{SF}}=-0.13+1.00 \log S_{0}^{\text {Chegsol }}, \mathrm{r}^{2}=0.90, \mathrm{~s}=0.52, \mathrm{~F}=1094, \mathrm{n}=125$. There is a slight bias: $S_{0}{ }^{\text {CheqSol }} / S_{0}{ }^{S F}=1.35$ (based on the intercept in the fit). The average SD $=0.25$, based on SF replicates, which is notably less than the $s=0.52$ from the correlation plot. The differences between the two sets of $\log S_{0}$ values were $\leq 0.25$ for $61 \%$ of the molecules. Using SF-based variances in a weighted linear regression analysis produced: $\log S_{0}{ }^{\mathrm{SF}}=-0.08+0.99 \log S_{0}^{\text {CheqSol }}$, with goodness-of-fit $=2.4$. It appears that the bias in the unweighted regression is influenced, in part, by the high variance of some of the SF values.

To put the above two comparisons into perspective, we selected a one-source set of SF distilled-water $S_{\mathrm{w}}$ measurements (with $\mathrm{pH}_{\mathrm{sat}}$ and $\mathrm{S}_{0}$ calculated with $\mathrm{pDISOL-X}$ ), performed in the same laboratory using the same assay protocol for 122 free-acids/-bases (Rytting et al. [24]). For 117 of the molecules, there were reported SF values from other laboratories. Figure 3c shows the SF-to-SF correlation plot. As expected, the two sets of data compared reasonably well: $\log S_{0}{ }^{S F}=-0.02+1.01 \log S_{0}^{\text {Rytting-SF }}, r^{2}=0.93, s=0.39, F=1637$, $\mathrm{n}=117$. The average $\mathrm{SD}=0.17$, based on non-Rytting SF replicates. (It is noteworthy that - with the exception of clofazimine - the Rytting set of molecules were relatively simple.) The differences between $\log \mathrm{S}_{0}$ values from the two SF sources were $\leq 0.25 \log$ unit for $70 \%$ of the molecules.

From the correlation plots in Figure 3, one may conclude that diverse sets of molecules show average reproducibility in the range 0.17 to 0.39 log unit by the "gold standard" shake-flask method. The highsolubility end of the range may be better determined, whereas the low-solubility end may have higher measurement errors. The expected reproducibility of the DTT method, $s=0.36$, appears to match that of the SF method, while the CheqSol method shows a slightly higher value, $\mathrm{s}=0.52$ (similar to the Katritzky et al. [67] estimate).

In the entire 4557-set, there were 786 replicate $\log S_{0}$ values from different laboratories, where two or more values could be averaged. The mean SD of all the averaged values is 0.19 log unit. It is expected that the mean value of 0.19 could be further reduced, to near 0.15 log unit, if corrections were applied to normalize $\log S_{0}$ values to a common temperature (e.g., $25^{\circ} \mathrm{C}$ ).

\section{Summary of the Factors Affecting Reproducibility}

Some of the factors affecting reproducibility of equilibrium solubility measurement discussed above may be summarized in the list:

- incomplete dissolution over the equilibration time (e.g., latent supersatured conditions) 
- inappropriate phase separation (e.g., first centrifuging a saturated solution, then filtering the supernatant)

- adsorption to the filter or assay vial surfaces

- poor wettability

- formations of drug aggregates/oligomers (dimers, trimers, ...), micelles, and drug-buffer complexes

- formation of ion-pair between ionic strength adjustor (e.g., $\mathrm{NaCl}, \mathrm{KCl}$, etc.) and charged form of drug

- polymorphs, hydrates, solvates, amorphous forms

- stereoisomers (DL-, D-, L-)

- cis-/trans- isomers

- not using buffers with low-soluble ionizable drugs

- not taking into account the effect of ambient $\mathrm{CO}_{2}$ on the water solubility of low-soluble bases

- using unnecessarily high buffer concentrations, possibly effecting drug-buffer complexation

- not measuring the final $\mathrm{pH}$ of the equilibrated saturated solution of ionizable drugs

- inadequate $\mathrm{pH}$ electrode calibration procedure at low and high $\mathrm{pH}$ and in salt solubility studies

- effect of impurities, especially those which are ionizable

- "promiscuous inhibitor" particles passing through filter

- compound instability at the extremes of pH or over long saturation times

- not sufficiently sensitive analytical methods used to determine very low drug concentration

From the perspective of predicting solubility, the impact of many of the above factors can be minimized by employing good experimental and data analysis practices. However, some of the factors leading to variability in measured solubility, such as the formation of polymorphs, hydrates, solvates, amorphous solids, and the impact of stereoisomers will be harder to deal with. Further in silico insights will be needed to address these challenges.

\section{Recommended Procedures for More Accurate Solubility-pH Measurements}

\section{Solubility Units, Tabulation of Results and the Use of Logarithmic Plots}

Following the format used in the Handbook of Aqueous Solubility Data (Yalkowsky et al. [19]), it is recommended that solubility be tabulated both in molarity and in practical $(\mathrm{mg} / \mathrm{mL})$ units. Standard deviations in the measured solubility (based on averaging three or more values) should be included in the table of values. Additionally, a graphical display of $\log S$ vs. $\mathrm{pH}$ (but not $S$ vs. $\mathrm{pH}$ ) would be visually helpful. In the logarithmic forms, the plots can serve as templates [52,57], to recognize the presence of aggregates, incomplete equilibration, corrections for the presence of small quantities of DMSO, etc.

\section{Solubility Methodology and the Benefits of Knowing the Accurate $p K a$}

The "gold standard" multiple-pH buffer shake-flask measurement is recommended for challenging ionizable molecules. Other methods may also be satisfactory, provided that the Henderson-Hasselbalch relationship is independently validated. Miniaturization can be recommended, as long as the protocols are rigorous and well validated.

For ionizable molecules, the measurement of $S_{\mathrm{w}}$ without stating the $\mathrm{pH}$ is not recommended. It is far better to measure $S_{\mathrm{pH}}$ values in well-qualified buffers (see below), at three or more $\mathrm{pH}$ values, bracketing the $\mathrm{p} K_{\mathrm{a}}$. 


\section{Ionization Constant}

The $\log S-\mathrm{pH}$ data should be evaluated to estimate the value of the intrinsic solubility, $S_{0}$. To do this, the independently-determined $\mathrm{p} K_{\mathrm{a}}$ is needed. It is sometimes very inaccurate to use the solubility- $\mathrm{pH}$ data to determine the $\mathrm{p} K_{\mathrm{a}}$, because usually the required assumption is that the Henderson-Hasselbalch equation is valid, but it may not be so for some particular low soluble molecule, or when high concentrations are used to characterize salt solubility. It is recommended that methods specifically designed to determine the ionization constants of very poorly soluble molecules are used (e.g., state-of-the-art UV spectrophotometry, capillary electrophoresis, potentiometry). These methods are widely available and have been fine-tuned for the challenge. Commercial $\mathrm{p} K_{\mathrm{a}}$ instruments based on the above three technologies are generally well-supported by their manufacturers.

\section{Temperature}

Solubility is a function of temperature, so the assay temperature always needs to be reported. It is clear that "room temperature" can be different from laboratory to laboratory, and in some cases, seasonally variable. It is advisable to measure and record the actual temperature in the sample vessel during the equilibration period. Or better yet, the measurement is performed in a thermostated vessel kept at 25/37 ${ }^{\circ} \mathrm{C}$.

\section{lonic Strength}

Measured solubility can be affected by ionic strength (particularly when salt solubility is measured), so the ionic strength usually needs to be reported. Not only do the ionic strength adjustor ( $\mathrm{NaCl}, \mathrm{KCl}$, etc.) and buffers contribute to the ionic strength, but so does the sample, especially when introduced in salt form.

\section{Equilibration Time and Stirring Protocol}

Finely granulated crystals dissolve more quickly than large crystals, illustrating the surface area effect in the classical Noyce-Whitney equation (Eq. 5 in the Appendix). Amorphous solids, which are generally more soluble than their crystalline counterparts, dissolve more quickly than crystals, illustrating the solubility effect - the rate of dissolution is proportional to the solubility (cf., Noyce-Whitney equation, Eq. 5 in the Appendix). Other examples of this are: anhydrous crystals dissolve more quickly than hydrates; usually pure enantiomers dissolve more quickly than racemic mixtures.

Vigorous stirring during the equilibration period can hasten the rate of dissolution, allowing for equilibrium to be reached more quickly (cf., simple Noyce-Whitney equation). However, for particle less than 1-5 $\mu \mathrm{m}$ in diameter, stirring speed has little influence on the rate of dissolution.

Towards the end of the equilibration period, stopping (or slowing) the stirring and allowing the solid to sediment probably contributes to formation of better quality crystals. The $80 \%$ stirring $-20 \%$ sedimentation timing protocol describe by Baka et al. [68] can be recommended.

In regions of $\mathrm{pH}$ where the sample molecule is largely ionized, equilibration times as short as 1-6 hours may be adequate. But for practically insoluble molecules, in $\mathrm{pH}$ regions where the molecule is largely in its uncharged form, equilibration times of $24-72 \mathrm{~h}$ may be required to reach equilibrium. Often, the conversion from less stable amorphous or anhydrous solids to more stable crystalline (often hydrate/solvate form) takes $12-24 \mathrm{~h}$, and sometimes longer.

In rigorous applications, the shake-flask method usually determines the equilibrium solubility of the most stable solid state form of the compound. Equilibration times as long as several days have been used. 
For example, terfenadine studies have used 3-4 d equilibration times. Steroids have been allowed to incubate for $2-12 \mathrm{~d}$. Probably 24 hours is a good average time, but it is a good idea to test longer times when measuring the equilibrium solubility of very poorly-soluble drugs: $48 \mathrm{~h}$, or even $3-7 \mathrm{~d}$. Perhaps special cases like amiodarone ( $30 \mathrm{~d}$ for full therapeutic effect of oral dose) may warrant long equilibration times. Ordinarily, such long times are not recommended.

\section{Shortening the Equilibration Time}

The CheqSol method uses cycles of dissolution and re-precipitation by $\mathrm{pH}$ adjustment to shorten the time to reach equilibrium. Presumably, the solid which re-precipitates in subsequent cycles is more active than the original crystalline material, perhaps being of small particle size (high surface area) and possibly amorphous.

Loftsson et al. [60] describes using temperature spiking cycles and seed crystal to hasten the equilibration period.

The Facilitated Dissolution Method of Higuchi, described in the Appendix can be used to speed up the rate of equilibration. The method is fundamentally rigorous and deserved much more attention than it has received. A comprehensive validation study would be welcomed.

\section{Solution Composition}

It is a good idea to keep the assay solutions simple and to define all components precisely!

When studying salt solubility, it is particularly helpful (and perhaps necessary) to specify the actual weight of sample in each vial. It is unhelpful to see statements such as "excess solid was added."

It is not enough to state that "water was used" as the solvent. Was there any added ionic strength adjustor (e.g., $0.15 \mathrm{M} \mathrm{NaCl}$, etc.)? Was carbon dioxide purged out? Low soluble bases such as terfenadine can indicate solubility over several magnitudes due to the effect of ambient $\mathrm{CO}_{2}$ in unbuffered solutions.

\section{pH Measurement using Glass Electrodes}

It is not a good practice to assume that the assay buffer $\mathrm{pH}$ is not altered by the addition of sample. It is highly recommended that the final $\mathrm{pH}$ of the saturated solution be carefully measured, using a properly standardized electrodes.

Particular attention should be given to the $\mathrm{pH}$ electrode calibration, especially when extreme $\mathrm{pH}$ is measured $(\mathrm{pH}<1$ or $\mathrm{pH}>12)$ or when the ionic strength reaches high values, as in the case of salt solubility measurement.

It is recommended that research-grade combination $\mathrm{pH}$ electrodes be used, with adequately described calibration procedure. Ideally, the four-parameter electrode calibration is recommended [55]. Those using the Sirius titrators will recognize the procedure as "Four-Plus" method, and those using the Pion titrators will recognize it as the "ABC" method. Simpler procedures may be satisfactory, as long as they are described precisely. The reported $\mathrm{pH}$ should be evidently either on the "operational" or the "concentration" scale; the "mixed" scale is not recommended [52].

A strong case can be made that general solubility equilibrium quotients are best formulated in the concentration scale (rather than activity), with pH electrodes standardized in a constant ionic medium, e.g., $0.15 \mathrm{M} \mathrm{NaCl}$ [52]. This may be especially important when salt solubility measurements are performed. 
How was $\mathrm{pH}$ adjusted (e.g., $0.1 \mathrm{M} \mathrm{HCl}, 1 \mathrm{M} \mathrm{NaOH}$ )? Statements like: "concentrated $\mathrm{H}_{3} \mathrm{PO}_{4}$ was added drop-wise to adjust the $\mathrm{pH}^{\prime \prime}$ are not clear enough. It is far better to state the precise concentration of the acid used. Record the precise volume of $\mathrm{pH}$ adjustor solution added. If one wishes to use ionizable titrants, such as phosphoric acid, acetic acid, maleic acid, tartaric acid, etc., it may be useful to add enough of the titrant so the starting $\mathrm{pH}$ is as low as desired in the assay. That way, the pre-acidified solution $\mathrm{pH}$ can be solely regulated by the amount of base titrant added. There is no real need to have a separate burette dedicated to the ionizable acid titrant. Result computation will be greatly simplified.

\section{Buffers}

Sufficient buffering is needed so that reliable $\mathrm{pH}$ can be measured and that the titrant can be dispensed precisely enough to adjust the solution to the desired $\mathrm{pH}$. Ordinarily, relatively low buffer concentrations (e.g., 5-10 mM) can be recommended. The sample itself may be an adequate buffer. It is not beneficial to overload the assay protocol with added complications arising from possible buffer-drug interactions. The use of universal buffers (e.g., Britton-Robinson, Sorensen, Mcllvaine) can be very useful, but it may also be a source of unintended/unnecessary complications when it comes to processing log $S-\mathrm{pH}$ data, due to drug-buffer interactions, particularly when salt solubility is measured. Some buffer components may cause difficulties in UV measurement. High concentration phosphate buffer is not recommended; however drug regulatory agencies may prescribe it.

It is inadequate to state that "buffers were used". Precisely which buffer compounds were used? Which salt forms of buffers were used? At what concentrations were they prepared? This information will be needed to calculate the total ionic strength. It may be a good idea to use zwitterionic buffers generally, or acid buffers with weak acid samples and basic buffers with base samples. Phosphate buffers interact with weak base drugs. So, caution is needed when phosphate buffers are used with low-soluble basic drugs. Purpose designed assays can better address the effect of phosphate anions on positively-charged sample molecules.

\section{Separating Solid from Saturated Solution}

Filtration can be recommended, using hydrophilic filters, typically 0.2-0.45 $\mu \mathrm{m}$ pore sizes. Chen et al. [58] found the hydrophilic PVDF (polyvinylidene fluoride) and PES (polyether sulfone) filters performing the best, and nylon the worst, in terms of excessive compound adsorption. It is useful to discard the first 10$25 \%$ filtered solution, to allow filters and surfaces to be saturated with adsorbed compound. The filtration step should not be rushed.

If "promiscuous inhibitors" (McGovern et al. [59]) are suspected, then the filtered solutions may be further subjected to ultracentrifugation. It is highly inadvisable to centrifuge first and then filter, for reasons discussed in the review.

\section{Conclusions}

Over 800 publications describing equilibrium solubility measurement of sparingly-soluble ionizable drug-like molecules by the shake-flask and related methods were examined. Many factors affecting the quality of the measurement were identified, and a number of suggestions were offered to improve the methodology. Some of the suggestions focused on improving methods for future measurements, and some referred to ways of extracting more reliable information from existing measurements. By normalizing data for $\mathrm{pH}$ (by using intrinsic solubility, $S_{0}$, derived from water solubility, $S_{\mathrm{w}}$ ) and temperature effects (by 
transforming measurements performed in the range $20-40{ }^{\circ} \mathrm{C}$ to the standard value of $25{ }^{\circ} \mathrm{C}$ ), it can be demonstrated that the expected interlaboratory reproducibility of $0.7 \mathrm{log}$ unit can be reduced to near 0.15 .

\section{Glossary}

DTT Dissolution Titration Template potentiometric method used to determine intrinsic solubility, $\mathrm{S}_{0}$

SF shake-flask method, the "gold standard" solubility measurement method

$\mathrm{HH} \quad$ Henderson-Hasselbalch equation (e.g., Eq. 1)

$K_{\mathrm{n}} \quad$ aggregation constant, where $\mathrm{n}$ is the degree of aggregation

$\mathrm{p} K_{\mathrm{a}} \quad$ negative logarithm of the ionization constant

$\mathrm{pH}_{\text {sat }}$ the equilibrium $\mathrm{pH}$ of a saturated solution

$S \quad$ solubility, ideally expressed in units of $\mathrm{mol} / \mathrm{L}(\mathrm{M}), \mu \mathrm{g} / \mathrm{mL}$, or $\mathrm{mg} / \mathrm{mL}$

$S_{0} \quad$ "intrinsic" solubility (i.e., the solubility of the uncharged form of the compound)

$S_{w} \quad$ "water" solubility, defined by dissolving enough pure free acid/base in distilled water (or water containing an inert salt - as ionic strength adjustor) to form a saturated solution. The final $\mathrm{pH}$ of the suspension, $\mathrm{pH}_{\text {sat }}$, and $\mathrm{S}_{0}$ can be calculated by the $\mathrm{HH}$ equation (when valid), provided the true $\mathrm{pK}_{\mathrm{a}}$ is known. Compound added as a salt form may disproportionate into free acid/base, depending on how much solid had been added. It is not generally possible to calculate the $\mathrm{pH}$ and $\mathrm{S}_{0}$ of such a drug salt suspension.

$S_{\mathrm{pH}} \quad$ "pH buffer" solubility (i.e., the total solubility of the compound at a well-defined $\mathrm{pH}_{\mathrm{sat}}$ )

\section{Acknowledgements}

Helpful discussions with Antonio Llinàs (AstraZeneca), Krisztina Takács-Novák and Gergely Völgyi (Semmelweis University, Budapest), Christel Bergström (Uppsala University), and Tatjana Verbić (University of Belgrade) are much appreciated. We are greatly indebted to Agustin G. Asuero (Univ. of Seville), Michael H. Abraham (University of London), and William E. Acree, Jr. (Univ. of N. Texas) for pointing out many important log S-pH publications. Uko Maran (Univ. of Tartu), Manfred Kansy, Holger Fischer, and Stefanie Bendels (Hoffman-La Roche) have provided valuable insights and leads into the literature of chemoinformatics, for which we are grateful.

\section{References}

[1] J. Huuskonen, M. Salo, J. Taskinen, J. Chem. Inf. Sci. 38 (1998) 450-456.

[2] J. Huuskonen, J. Rantanen, D. Livingstone, Eur. J. Med. Chem. 35 (2000) 1081-1088.

[3] M.H. Abraham, J. Le, J. Pharm. Sci. 88 (1999) 868-880.

[4] W.L. Jorgensen, E.M. Duffy, Bioorg. Med. Chem. Lett. 10 (2000) 1155-1158.

[5] W.L. Jorgensen, E.M. Duffy, Adv. Drug Deliv. Rev. 54 (2002) 355-366.

[6] C.A.S. Bergström, U. Norinder, K. Luthman, P. Artursson, Pharm. Res. 19 (2002) 182-188.

[7] T.J. Hou, K. Xia, W. Zhang, X.J. Xu, J. Chem. Inf. Comput. Sci. 44 (2004) 266-275.

[8] J.S. Delaney, Drug Discov. Today 10 (2005) 289-295.

[9] J.C. Dearden, Expert Opin. Drug Discov. 1 (2006) 31-52.

[10] K.V. Balakin, N.P. Savchuk, I.V. Tetko, Curr. Med. Chem. 13 (2006) 223-241.

[11] J. Taskinen, U. Norinder, In silico predictions of solubility. In: B. Testa, H. van de Waterbeemd (eds.). Comprehensive Medicinal Chemistry II, Elsevier: Oxford, UK, 2007, pp. 627-648. 
[12] P. Jain, S.H. Yalkowsky, Int. J. Pharm. 385 (2010) 1-5.

[13] A. Shayanfar, A. Jouyban, Lat. Am. J. Pharm. 30 (2011) 1525-1530.

[14] J. Wang, T. Hou, Combi. Chem. High Throughput Scr. 14 (2011) 1-11.

[15] D. Elder, R. Holm, Int. J. Pharm. 453 (2013) 3-11.

[16] J.L. McDonagh, N. Nath, L. De Ferrari, T. Van Mourik, J.B. Mitchell, J. Chem. Inf. Model. 54 (2014) 844-856.

[17] D.S. Palmer, J.B.O. Mitchell, Mol. Pharmaceutics 11 (2014) 2962-2972.

[18] B. Faller, P. Ertl, Adv. Drug Deliv. Rev. 59 (2007) 533-545.

[19] S.H. Yalkowsky, Y. He, P. Jain, Handbook of Aqueous Solubility Data, Second Edition. CRC Press, Boca Raton, FL, 2010.

[20] P. Howard, W. Meylan, PHYSPROP DATABASE. Syracuse Research Corp., Environmental Science Center, N. Syracuse, NY, Sept. 1999.

[21] Syracuse Research Corporation. The Physical Properties Database (PHYSPROP) http://www.srcinc.com/what-we-do/environmental/scientific-databases.html (accessed 18 Feb 2015).

[22] Analytical Profiles of Drug Substances (Analytical Profiles of Drug Substances and Excipients; Profiles of Drug Substances, Excipients and Related Methodology). K. Florey (ed., vols. 1-20),H.G. Brittain (ed., vols. 21-39). Academic Press, San Diego, 1972-2014.

[23] J.W. McFarland, A. Avdeef, C.M. Berger, O.A. Raevsky, J. Chem. Inf. Comput. Sci. 41 (2001) 13551359.

[24] E. Rytting, K.A. Lentz, X.Q. Chen, F. Qian, S. Venkatesh, Pharm Res. 21 (2004) 237-244.

[25] C.A.S. Bergström, C.M. Wassvik, U. Norinder, K. Luthman, P. Artursson, J. Chem. Inf. Comput. Sci. 44 (2004) 1477-1488.

[26] A. Llinàs, R.C. Glen, J.M. Goodman, J. Chem. Inf. Model. 48 (2008) 1289-1303.

[27] A.J. Hopfinger, E.X. Esposito, A. Llinàs, R.C. Glen, J.M. Goodman, J. Chem. Inf. Model. 49 (2009) 1-5.

[28] W.P. Walters, What are our models really telling us? A practical tutorial on avoiding common mistakes when building predictive models. In: J. Bajorath (Ed.). Chemoinformatics for Drug Discovery. John Wiley \& Sons, Hoboken, NJ, 2014, pp. 1-31.

[29] G. Landrum, R. Lewis, A. Palmer, N. Stiefl, A. Vulpetti, J. Cheminform. 3 (2011) 1-1.

[30] M.H. Abraham, A. Ibrahim, A.M. Zissimos, Y.H. Zhao, J. Comer, D.P. Reynolds, Drug Disc. Today 7 (2002) 1056-1063.

[31] A. SID Lang, J-C Bradley, ONS Melting Point Model 010. QDB archive, DOI: 10.15152/QDB.104. QsarDB content. Property mpC.

[32] G. Völgyi, A. Marosi, K. Takács-Novák, A. Avdeef, ADMET \& DMPK 1(4) (2013) 48-62.

[33] A. Avdeef, ADMET \& DMPK 2(1) (2014) 33-42.

[34] A. Avdeef, ADMET \& DMPK 2(1) (2014) 43-55.

[35] M. Stuart, K. Box, Anal. Chem. 77 (2005) 983-990.

[36] C. Sköld, S. Winiwarter, J. Wernevik, F. Bergström, L. Engström, R. Allen, L. Box, J. Comer, J. Mole, A. Hallberg, H. Lennernäs, T. Lundstedt, A-L. Ungell, A. Karlén, J. Med. Chem. 49 (2006) 6660-6671.

[37] A. Llinàs, J.C. Burley, K.J. Box, R.C. Glen, J.M. Goodman, J. Med. Chem. 50 (2007) 979-983.

[38] K.J. Box, J.E.A. Comer, Curr. Drug Metab. 9 (2008) 869-878.

[39] L.Y.S. Narasimham, V.D. Barhate, J. Pharmacy Res. 4(2) (2011) 532-536.

[40] Y.-L. Hsieh, G.A. Ilevbare, B. van Eerdenbrugh, K.J. Box, M.V. Sanchez-Felix, L.S. Taylor, Pharm. Res. 29 (2012) 2738-2753.

[41] J. Comer, S. Judge, D. Matthews, L. Towes, B. Falcone, J. Goodman, J. Dearden, ADMET \& DMPK 2(1) (2014) 18-32.

[42] D. Schönherr, U. Wollatz, D. Haznar-Garbacz, U. Hanke, K.J. Box, R. Taylor, R. Ruiz, S. Beato, D. 
Becker, W. Weitschies, Eur. J. Pharm. Biopharm. 92 (2015) 155-170.

[43] A. Avdeef, Pharm. Pharmacol. Commun. 4 (1998) 165-178.

[44] A. Avdeef, C.M. Berger, C. Brownell, Pharm. Res. 17 (2000) 85-89.

[45] A. Avdeef, C.M. Berger, Eur. J. Pharm. Sci. 14 (2001) 281-291.

[46] A. Avdeef, Curr. Topics Med. Chem. 1 (2001) 277-351.

[47] B. Faller, F. Wohnsland, Physicochemical parameters as tools in drug discovery and lead optimization. In: B. Testa, H. van de Waterbeemd, G. Folkers, R. Guy, R. (Eds.). Pharmacokinetic Optimization in Drug Research. Verlag Helvetica Chimica Acta: Zürich and Wiley - VCH: Weinheim, pp. 257-274 (2001).

[48] C.A.S. Bergström, M. Strafford, L. Lazarova, A. Avdeef, K. Luthman, P. Artursson, J. Med. Chem. 46 (2003) 558-570.

[49] A.F. Fioritto, S.N. Bhattachar, J.A. Wesley, Int. J. Pharm. 330 (2007) 105-113.

[50] G. Ottaviani, D.J. Gosling, C. Patissier, S. Rodde, L. Zhou, B. Faller, Eur. J. Pharm. Sci. 41 (2010) 452457.

[51] Y. Surakitbanharn, R. McCandless, J.P. Krzyzaniak, R-M. Dannenfelser, S.H. Yalkowsky, J. Pharm. Sci. 84 (1995) 720-723.

[52] A. Avdeef, Absorption and Drug Development Second Edition. Wiley-Interscience: New York. 2012.

[53] E. Shoghi, E. Fuguet, E. Bosch, C. Rafols, Eur. J. Pharm. Sci. 48 (2012) 290-300.

[54] I. Zimmermann, Int. J. Pharm. 31 (1986) 69-74.

[55] A. Avdeef, J.J. Bucher, Anal. Chem. 50 (1978) 2137-2142.

[56] Z. Wang, L.S. Burrell, W.J. Lambert, J. Pharm. Sci. 91 (2002) 1445-1455.

[57] A. Avdeef, Adv. Drug Deliv. Rev. 59 (2007) 568-590

[58] T-M. Chen, H. Shen, C. Zhu, Combi. Chem. HTS 5 (2002) 575-581.

[59] S.L. McGovern, E. Caselli, N. Grigorieff, B.K. Shoichet, J. Med. Chem. 45 (2002) 1712-1722.

[60] T. Loftsson, D. Hreinsdóttir, M. Másson, Int. J. Pharm. 302 (2005) 18-28.

[61] S. Venkatesh, J. Li, Y. Xu,R. Vishnuvajjala, Pharm. Res. 13 (1996) 1453-1459.

[62] Y. He, S.E. Tabibi, S.H. Yalkowsky, J. Pharm. Sci. 95 (2006) 97-107.

[63] A. Fini, G. Fazio, G. Feroci, Int. J. Pharm. 126 (1995) 95-102.

[64] M. Pudipeddi, A.T.M. Serajuddin, J. Pharm. Sci. 94 (2005) 929-939.

[65] B.D. Anderson, K.P. Flora, Preparation of water-soluble compounds through salt formation. In: C.G. Wermuth, (Ed.). The Practice of Medicinal Chemistry. Academic Press, London, pp. 739-754 (1996).

[66] H. Kishi, Y. Hashimoto, Chemosphere 18 (1989) 1749-1759.

[67] A.R. Katritzky, Y. Wang, S. Sild, T. Tamm, M. Karelson, J. Chem. Inf. Comput. Sci. 38 (1998) 720-725.

[68] E. Baka, J.E.A. Comer, K. Takács-Novák, J. Pharm. Biomed. Anal. 46 (2008) 335-341.

[69] A. Avdeef, D. Voloboy, A. Foreman, Dissolution and solubility. In: B. Testa, H. van de Waterbeemd (eds.). Comprehensive Medicinal Chemistry II, Elsevier: Oxford, UK, 2007, pp. 399-423.

[70] A. Avdeef, O. Tsinman, Pharm. Res. 25 (2008) 2613-2627.

[71] K. Tsinman, A. Avdeef, O. Tsinman, D. Voloboy, Pharm. Res. 26 (2009) 2093-2100.

[72] A. Avdeef, K. Tsinman, O. Tsinman, N. Sun, D. Voloboy, Chem. Biodiv. 11 (2009) 1796-1811.

[73] J.H. Fagerberg, O. Tsinman, K. Tsinman, N. Sun, A. Avdeef, C.A.S. Bergström, Mol. Pharmaceutics 7 (2010) 1419-1430.

[74] A. Avdeef, S. Bendels, O. Tsinman, M. Kansy, Pharm. Res. 24 (2007) 530-545.

[75] J. Alsenz, M. Kansy, Adv. Drug Deliv. Rev. 59 (2007) 546-567.

[76] J. Alsenz, E. Meister, E. Haenel, J. Pharm. Sci. 96 (2007) 1748-1762.

[77] C.A.S. Bergström, K. Luthman, P. Artursson, Eur. J. Pharm. Sci. 22 (2004) 387-398. 
[78] A. Glomme, J. März, J.B. Dressman, J. Pharm. Sci., 94 (2005) 1-16.

[79] T. Higuchi, F.M. Shih, T. Kimura, J.H. Rytting, J. Pharm. Sci. 68 (1979) 1267-1272.

[80] W.H. Streng, S.K. His, P.E. Helms, H.G.H. Tan, J. Pharm. Sci. 73 (1984) 1679-1684.

[81] F. Wohnsland, B. Faller, J. Med. Chem. 44 (2001) 923-930.

[82] G. Völgyi, E. Baka, K.J. Box, J.E.A. Comer, K. Takács-Novák, Anal.Chim. Acta 673 (2010) 40-46. 


\section{Appendix - Brief Summary of Methods Suitable for Measuring Equilibrium Solubility}

The Appendix briefly describes methods which can potentially be used to generate intrinsic solubility of sparingly-soluble drugs. Kinetic methods are not suitable for such application, and are thus not considered.

\section{Classical Saturation Shake-Flask (SF) - Still the 'Gold Standard'}

Solubility measurement as a function of $\mathrm{pH}$ under equilibrium conditions usually requires long equilibration times ( $12 \mathrm{~h}-7 \mathrm{~d}$ ). The shake-flask (SF) method is a relatively simple procedure: the drug is added as solid to a standard buffer solution (in a flask) until saturation occurs, indicated by excess undissolved drug. The thermostated saturated solution is shaken as the suspension equilibrates. After filtration or centrifugation, the concentration of the substance in the supernatant solution is then determined using HPLC, usually with UV detection. For a solubility-pH profile, parallel measurements need to be performed in several different pH buffers. Baka et al. [68] suggested concrete ways to improve the quality of the SF measurements. In high-quality SF measurements, (a) it is actually verified that the dissolution/precipitation of the solid has reached equilibrium, (b) the final (equilibrium) $\mathrm{pH}$ is measured, and (c) the equilibrated solid is isolated and identified (or characterized). The measured solubility is expected to be that of the most stable polymorph, which can often be a hydrate.

\section{Micro-Dissolution for Polymorph/Solvate Solubility Measurement ( $\mu$ DISS Profiler)}

By USP standards, dissolution equipment typically uses $900-\mathrm{mL}$ solution volumes, so it would be impractical to determine solubility of drugs, since an excessive amount of compound would be required to form a saturated solution. However, the miniaturized-volume dissolution apparatus, $\mu$ DISS Profiler (Pion Inc.), uses $1.0 \mathrm{~mL}$ working volumes and makes it practical to determine solubility (Avdeef et al. [69]; Avdeef and Tsinman [70]; Tsinman et al. [71]; Avdeef et al. [72]; Fagerberg et al. [73]). Since the instrument uses in situ fiber optic UV (diode array) detection, the progression of dissolution may be followed in real time, making it possible to characterize the (transient) solubility of different polymorphs/solvates, over periods of days, if necessary. It has been demonstrated that 10-100 $\mu$ g of powder can be characterized for both the transient polymorph solubility and equilibrium solubility of the most stable form of the solid. A hydroxypropyl-6-cyclodextrin phase-solubility study of itraconazole using the micro-dissolution apparatus estimated the active polymorph solubility of itraconazole to be $<9 \mathrm{ng} / \mathrm{mL}$.

\section{Equilibrium 96-Well Microtitre Plate Methods}

To increase throughput and decrease sample consumption, several pharmaceutical companies have transferred the larger-volume classical SF method to smaller-volume 96-well microtitre plate format linked to robotic liquid handling systems, using several distinct approaches (including those which avoid use of DMSO). There are trade-offs in the high-throughput methods, and usually, the quality of the data are not expected to be as accurate as those obtained by the SF method.

Since the compound is usually introduced as aliquots of a $10 \mathrm{mM}$ DMSO stock solution, such methods have an upper solubility limit, typically less than $150 \mu \mathrm{M}$. Also, the presence of the small amount of DMSO in the final buffer solution increases the measured solubility values of the most insoluble compounds by up to 100 -fold (e.g., glibenclamide, clotrimazole), compared to DMSO-free conditions. This appears to be highly compound-dependent (Chen et al. [58]). Quality results require this DMSO-effect to be factored out (e.g., pDISOL-X method). 
For acceptably reliable aqueous intrinsic solubility $\left(S_{0}\right)$ to be determined from single-pH determination (of compounds introduced from DMSO stock solutions), it is necessary (a) to remove the DMSO originating from the stock solution, (b) to know the $\mathrm{p} K_{\mathrm{a}}$ value of the test compound, and (c) to assume that the Henderson-Hasselbalch $(\mathrm{HH})$ equation is a valid description of the $\log S-\mathrm{pH}$ relationship.

If the measurement is performed in the presence of $\leq 1 \% \mathrm{v} / \mathrm{v}$ DMSO, there is a way to determine $S_{0}$, provided that the solubility measurement is done at several values of $\mathrm{pH}$ over a wide enough a $\mathrm{pH}$ range ([52]; $p$ DISOL-X method). If the $\mathrm{p} K_{\mathrm{a}}$ is known, then it is not necessary to assume that the $\mathrm{HH}$ equation holds, as has been demonstrated by Avdeef et al. [74].

\section{Lyophilization Method ('GeneVac' 96-Well DMSO-removing Device)}

Some pharmaceutical companies have implemented methods where aliquots of $10 \mathrm{mM}$ DMSO stock solutions of test compounds are added to a microtitre plate. Then, the DMSO is removed by lyophilization (e.g., using GeneVac vacuum centrifugation apparatus), after which, a pH 6.5 or 7.4 buffer is added to the compound residues in the microtitre plate. The plate is sealed and allowed to incubate up to $24 \mathrm{~h}$, usually at room temperature, during which time the plate may be shaken. For best results, the final (equilibrium) $\mathrm{pH}$ needs to be measured. With the initial DMSO removed, measured solubility values can match those obtained by the traditional SF method. Alsenz and Kansy [75] described the Lyophilization Solubility Assay (LYSA) based on UV plate reader and HPLC methods.

\section{Equilibrium Solubility Assay (DMSO-free 'THESA' Method)}

Alsenz and Kansy [75] also described the Thermodynamic Solubility Assay (THESA) microtitre plate method where $<1 \mathrm{mg}$ of weighed solid sample is added a pH 6.5 buffer solution. The suspensions are stirred for $2 \mathrm{~h}$ and allowed to sit for another $20 \mathrm{~h}$, before filtration. A provision for reading the final $\mathrm{pH}$ is included in the protocol. The advantage of the THESA method is that the compound is introduced in the original solid state.

\section{Partially-Automated Solubility Screening (DMSO-free 'PASS' Method)}

Alsenz et al. [76] described the microtitre plate Partially-Automated Solubility Screening (PASS), where solid compounds (DMSO-free) are suspended as slurries in volatile heptane, sonicated to increase fractionation and dispersion, and then dispensed quickly in small aliquots into microtitre plate wells. From the dispensed volume, the weight of drug is calculated. The advantage of the PASS method is that the compound is introduced in the original solid state. The heptane is allowed to evaporate, before buffer is added. This procedure inherently can be used to assess relatively high solubility (several $\mathrm{mg} / \mathrm{mL}$ ) by a microtitre plate method. The comparison of PASS solubility values to those from standard SF values was good.

\section{Small-Scale Shake-Flask Method (SSF)}

Bergström et al. [6] described the Small-Scale Shake-Flask (SSF) method, where crystalline compounds were added to $50-200 \mu \mathrm{L}$ solutions whose $\mathrm{pH}$ was then adjusted to at least one $\mathrm{pH}$ below the $\mathrm{p} K_{\mathrm{a}}$ for acids and one $\mathrm{pH}$ above the $\mathrm{p} K_{\mathrm{a}}$ for bases, in an effort to measure $S_{0}$ directly. After 24-72 $\mathrm{h}$ of stirring, the solutions were centrifuged to separate the phases. Seventeen compounds were measured $(0.7 \mathrm{ng} / \mathrm{mL}$ to 6 $\mathrm{mg} / \mathrm{mL}$ ). Expanding the SSF approach, Bergström et al. [77] further studied 25 bases over a wide $\mathrm{pH}$ range, using $0.15 \mathrm{M}$ phosphate buffer adjusted with $\mathrm{KOH}$ or $\mathrm{H}_{3} \mathrm{PO}_{4}$. The suspensions were incubated for $24 \mathrm{~h}$. Phases were separated by centrifugation. An in-depth analysis of the 25 bases has been recently reported 
[34], where it was tentatively suggested that $24 \mathrm{~h}$ may not be sufficient equilibration time for several of the sparingly-soluble bases studied. Other concerns about pH control were raised by Völgyi et al. [82].

\section{Miniaturized Shake-Flask (MSF)}

Glomme et al. [78] described the Miniaturized Shake-Flask (MSF) method. The sample is weighed (twice the in silico-estimated solubility) directly into the Whatman UniPrep filter chamber, which is then filled with $2 \mathrm{~mL}$ of buffer solution. The capped chambers are agitated for $24 \mathrm{~h}$ at $37^{\circ} \mathrm{C}$. Afterwards, a chambermated plunger equipped with a filter is pushed over the sample solution to separate the solid. The final $\mathrm{pH}$ is read at the end of the equilibration period.

\section{Dual-Phase Potentiometric Methods}

The two related potentiometric methods described below are only suitable for ionizable compounds, whose $\mathrm{pK}_{\mathrm{a}}$ can be determined in situ (DTT) or need to be determined in a separate method (CheqSol).

\section{Dissolution Template Titration Method (DTT)}

The Dissolution Template Titration (DTT) semi-automated intrinsic solubility potentiometric method [43] takes the estimated $\mathrm{p} K_{\mathrm{a}}$ and the octanol/water partition coefficient, $\log P_{\mathrm{OCT}}$, as input parameters to predict $S_{0}$. The DTT procedure then simulates the entire titration curve before the assay starts. This defines a "template" for data collection. Titration is done in the direction of increasing dissolution, taking the saturated solutions past the point of complete dissolution. The Noyes-Whitney equation (Eq. 5 below) is used to set the pace of data collection. The more insoluble the compound is (based on the template), the longer is the assay time (typically, $3-10 \mathrm{~h}$ ).

Data are analyzed using Bjerrum plots: $\bar{n}_{H}$, the average number of bound protons, versus $\mathrm{pH}$. Since it is known how much strong acid, $[\mathrm{HCl}]$, and strong base, $[\mathrm{KOH}]$, have been added to the solution at any point and since it is known how many dissociable protons, $\mathrm{n}$, the sample adds to the solution, the total hydrogen ion concentration is defined. The difference between the latter and the free hydrogen $(\mathrm{pH}$ electrode reading) concentrations is equal to the concentration of the bound hydrogen, which, when divided by the sample concentration, $C$, results in $\overline{\mathrm{n}}_{\mathrm{H}}=\left\{[\mathrm{HCl}]-[\mathrm{KOH}]+\mathrm{nC}-\left[\mathrm{H}^{+}\right]+\left[\mathrm{OH}^{-}\right]\right\} / \mathrm{C}$.

The $\mathrm{pH}$ at half-integral value of $\overline{\mathrm{n}}_{\mathrm{H}}$ equals $\mathrm{p} K_{\mathrm{a}}^{\mathrm{APP}}$, the apparent $\mathrm{p} K_{\mathrm{a}}$. The presence of precipitate is indicated by the $\mathrm{p} K_{\mathrm{a}}{ }^{\mathrm{APP}}$ shifting to a higher value for acids and to a lower value for bases, compared to the true $\mathrm{p} K_{\mathrm{a}}$. The intrinsic solubility can be deduced by inspection of the curves and applying the relationship $\log S_{0}=\log (C / 2)-\left|p K_{\mathrm{a}}^{A P P}-\mathrm{p} K_{\mathrm{a}}\right|$. The graphically-estimated constant is subsequently refined by a massbalance weighted nonlinear regression procedure, which does not require that the Henderson-Hasselbalch relationship be valid (although, often, it is assumed to be so). The $\mathrm{p} K_{\mathrm{a}}$ can be determined alongside intrinsic solubility.

\section{CheqSol Potentiometric Method}

Stuart and Box [35] embraced the $\overline{\mathrm{n}}_{\mathrm{H}}$ part of the DTT method and developed a valuable novel way to speed up the time to reach equilibration. Focusing on the $\mathrm{pH}$ region where $\overline{\mathrm{n}}_{\mathrm{H}}$ is expected to be near halfintegral, the CheqSol instrument quickly dissolves the solid by raising the $\mathrm{pH}$ for acids (e.g., to 10-12) or lowering the $\mathrm{pH}$ for bases (e.g., to 1-2). Afterwards, the original $\mathrm{pH}$ is re-established by adding acid/base titrant, whereupon the solid re-precipitates, possibly as an amorphous phase. The procedure of dissolution-precipitation is cycled several times, from which the $\mathrm{pH}$ corresponding to a known value of $\mathrm{n}_{\mathrm{H}}$ (near 0.5 ) is determined by interpolation. Using the DDT $\mathrm{n}_{H}$ relationships, the intrinsic $S_{0}$ is calculated from 
a single $\mathrm{pH}$ point, suggested by the value of the $\mathrm{p} K_{\mathrm{a}}$. Since the method is not based on the general massbalance base regression analysis, CheqSol implicitly assumes that the Henderson-Hasselbalch equation is always valid, which in some cases could potentially be a source of systematic error.

\section{Facilitated Dissolution Method (FDM)}

The Facilitated Dissolution Method (FDM), devised by Higuchi et al. [79], can be used to overcome extremely low equilibration rates during solubility measurement of practically-insoluble compounds. Since it is rarely practiced, the method is described here in some detail (cf., [52]). In the FDM approach, a twofold excess of solid over that needed to saturate the solution is recommended. To overcome the expected slow dissolution, a small volume of an immiscible organic solvent (e.g., $2 \% \mathrm{v} / \mathrm{v}$ hexadecane) is added to the aqueous solution of the sparingly-soluble compound.

As long as the saturated system contains three distinct phases (solid, oil, and water), the solubility value is not altered by the presence of the water-immiscible oil. (This is sufficiently but not precisely true for ionizable compounds, as commented below.) To show this, consider a weak base example (e.g., terfenadine), for which the FDM equilibrium reactions are:

$$
\begin{array}{ll}
\mathrm{B}(\mathrm{s}) \leftrightarrow \mathrm{B} \text { (org) } & S_{\mathrm{ORG}}=[\mathrm{B}(\text { org })] \\
\mathrm{B} \leftrightarrow \mathrm{B}(\text { org }) & P_{\mathrm{O} / \mathrm{W}}=[\mathrm{B}(\text { org })] /[\mathrm{B}]
\end{array}
$$

where Eq. 2 denotes the solubility of the compound in oil, $S_{\mathrm{ORG}}$, and Eq. 3 denotes the oil-water partition coefficient of the compound, $P_{\mathrm{O} / \mathrm{w}}$. By subtracting the partition reaction from the solubility-in-oil reaction, one gets the expected solubility-in-water equation,

$$
\mathrm{B}(\mathrm{s}) \leftrightarrow \mathrm{B} \quad \mathrm{S}_{\mathrm{W}}=\mathrm{S}_{\mathrm{ORG}} / \mathrm{P}_{\mathrm{O} / \mathrm{W}}
$$

So, the presence of a small quantity of oil, into which the water sparingly-soluble compound can appreciably dissolve, does not affect the aqueous solubility value. According to the Noyes-Whitney dissolution rate $\left(\mu \mathrm{g} / \mathrm{cm}^{3} \cdot \mathrm{s}\right)$ equation under sink condition,

$$
\mathrm{d}[\mathrm{B}] / \mathrm{d} t=(A / V) P_{\mathrm{ABL}} S_{\mathrm{W}}
$$

where $A=$ powder surface area $\left(\mathrm{cm}^{2}\right), V=$ volume of aqueous solution $\left(\mathrm{cm}^{3}\right), P_{\mathrm{ABL}}=$ permeability of the aqueous boundary layer adjacent to the surface of the solid particles $(\mathrm{cm} / \mathrm{s})$, and $S_{\mathrm{W}}=$ aqueous solubility $\left(\mu \mathrm{g} / \mathrm{cm}^{3}\right)$.

The addition of the oil may also alleviate problems due to poor wettability of the original crystalline solid.

Let's consider the terfenadine FDM example explicitly. The intrinsic solubility of the weak base in water is $5.6 \times 10^{-8} \mathrm{M}\left(\log \mathrm{S}_{0}=-7.25\right)$ at $25^{\circ} \mathrm{C}$ (Streng et al. [80]; data analyzed using $\left.p D I S O L-X\right)$. The measured $p K_{\mathrm{a}}$ is 9.91 at $25{ }^{\circ} \mathrm{C}, I=0.15 \mathrm{M}$ (Origin-shifted Yasuda-Shedlovsky method [52]). Consider $20 \mu \mathrm{g}$ of terfenadine free base added to $1 \mathrm{~mL}$ of $10 \mathrm{mM}$ taurine buffer in $0.15 \mathrm{M} \mathrm{NaCl}$ at pH 9.0, containing $20 \mu \mathrm{L}$ of hexadecane. Let's assume $100 \mu \mathrm{m}$ (diam.) spherical particles of solid in the suspension at the start. The hexadecanewater partition coefficient of terfenadine is estimated to be $\log P_{\mathrm{HXD} / \mathrm{W}}=2.3$ (using calibration standards from Wohnsland and Faller [81]). The dissolution simulation feature of $p D I S O L-X$ was used to calculate the precise concentrations and quantities in each of the three phases. At $\mathrm{pH} 9.0,0.302 \mu \mathrm{g}$ of the drug is predicted to dissolve in the buffer in the absence of added hexadecane, with $19.70 \mu \mathrm{g}$ remaining in the solid state. The calculated total surface area of the solid is initially $0.0086 \mathrm{~cm}^{2}$ and decreases by $1.3 \%$ at saturation. The $(A / V)$ factor for Eq. 5 is $0.0086 \mathrm{~cm}^{-1}$. After the addition of $20 \mu \mathrm{L}$ of hexadecane, the 
calculated amount of the drug in water is still exactly $0.302 \mu \mathrm{g}$; the amount in the solid is now $19.59 \mu \mathrm{g}$, as $0.20 \mu \mathrm{g}$ of the drug partitions into the $20 \mu \mathrm{L}$ of hexadecane. The solubility in hexadecane is calculated to be $2.2 \times 10^{-7} \mathrm{M}$, compared to $6.4 \times 10^{-7} \mathrm{M}$ in the buffer. The smaller volume of the oil phase increase the rate of dissolution into the oil phase: the $(\mathrm{A} / \mathrm{V})$ factor between the solid surface and the oil volume is 0.0086 $\mathrm{cm}^{2} / 0.02 \mathrm{~cm}^{3}=0.43 \mathrm{~cm}^{-1}$. This suggests that the rate of dissolution into the oil phase will be about 50 times greater than the rate of dissolution into the water phase. What about the rate of transfer from the oil phase into water (to complete the transfer cycle in Eq. 4)? This is expected to be high in a well stirred (e.g., 500 RPM) solution, dispersing the oil into tiny droplets, thus increasing oil-water interfacial surface area. Hence, a small amount of adjunct oil substantially increases the overall dissolution rate associated with Eq. 5 , without affecting the equilibrium solubility of terfenadine.

It is important to point out that the FDM approach does not work for ionizable compounds in poorlybuffered solutions, and generally cannot be applied in the DTT or the CheqSol method (since buffers are not generally used). Using the pDISOL-X program, simulations in buffer-free solutions show that the addition of $10 \mu \mathrm{L}$ hexadecane can affect the water solubility of terfenadine, through a subtle interplay of the various $\mathrm{pH}$-dependent equilibria. The effect is lessened if a very large excess of terfenadine were added. But this is not recommended, as discussed by Higuchi et al. [79].

As far as we are aware, the FDM approach was last applied by Venkatesh et al. [61] in the solubility determination of cosalane, a steroid derivative with aqueous intrinsic solubility of much less than $1 \mathrm{ng} / \mathrm{mL}$. The $\mathrm{pK}_{\mathrm{a}}$ values of cosalane have not been reported to date, so there may be some uncertainty in the intrinsic solubility of cosalane. Since the FDM method is best applied to practically-insoluble molecules, probably LC/MS-MS resources would be required to measure the extremely low sample concentrations in saturated solutions.

C 2015 by the authors; licensee IAPC, Zagreb, Croatia. This article is an open-access article distributed under the terms and conditions of the Creative Commons Attribution license (http://creativecommons.org/licenses/by/3.0/) (cc) $\mathrm{Er}$ 